\title{
Rheology for thickened tailings and paste — history, state-of-the-art and future directions
}

\author{
F. Sofrà Rheological Consulting Services Pty Ltd, Australia \\ D.V. Boger Monash University; and The University of Melbourne, Australia
}

\begin{abstract}
A significant development during the evolution of thickened tailings and paste technology has been the increased understanding of the importance of rheology for both design and operation of thickened tailings and paste systems. It is now well accepted that dewatering, pipeline transport and deposition processes are all rheology governed and that the more the rheology is understood, and in some cases manipulated, the more successful the operation will be. This increased knowledge and adoption of rheology has led to a paradigm shift where tailings are engineered to suit the environment rather than engineering the environment to suit the tailings.

In the early to mid 1970s when Eli Robinsky first introduced the concept of thickened tailings disposal, the systems were based primarily on empirical flume tests. Since that time, numerous advances in measurement techniques have facilitated the understanding of the influence of variables such as particle size distribution and shape, shear effects (rate and time of shear) and mineralogy on the rheology of tailings. Compressional rheology has also emerged as a whole new area of interest. This progress, coupled with advances in understanding the interrelationship between surface chemistry and rheology, has highlighted methods of exploiting and/or manipulating the dewatering, flow and depositional properties of tailings and backfill. As flocculant and other additive technologies advance, we may begin to see more complex rheological phenomena and new measurement techniques may need to be adopted or developed for a fuller understanding and exploitation of slurry and paste rheology. The paper examines the driving issues faced in the past, now and likely in the future, the testwork that has been developed and its applicability or limitations and how this has progressed thickened tailings and paste technology.
\end{abstract}

\section{Introduction}

Thickened tailings and paste technology is now well accepted as one of the options available for storage and disposal of mining and mineral processing wastes. However, this has not always been the case. In the 1970s, Eli Robinsky (Robinsky, 1975) developed the concept of dewatering tailings to a higher concentration and depositing to form a self supporting slope without the need for traditional settlement ponds and high perimeter dams. Though in theory there were benefits to this approach including a potentially smaller footprint, water and reagent savings and environmental benefits such as increased stability and enhanced reclamation, the concept was not widely implemented by the industry for many years.

Some of the reasons for the slow implementation of thickened tailings and paste systems include the difficulty in thickening tailings sufficiently, the difficulty in pumping high solids content slurries and a lack of understanding of slurry behaviour upon deposition, not to mention the additional capital expenditure. In general, there was a lack of understanding of how increasing the solids concentration of slurries changed the dewatering and flow behaviour and the factors contributing to these changes in behaviour.

The current knowledge in the area of concentrated slurry systems is much greater than in the 1970s and this has facilitated increased implementation of thickened tailings and paste systems. Rheology is one area that has made a very significant contribution, however, even at the early Paste and Thickened Tailings Seminars in 1999 and 2000, rheology was barely mentioned. Since that time, the development and, more importantly, implementation of various rheological measurement techniques has contributed a great deal to the understanding of concentrated slurry behaviour and has facilitated advancements in many areas of slurry management for thickened tailings and paste. This paper focuses on the advancement of the implementation 
of rheological techniques, summarises the current situation and suggests some areas where rheology may be instrumental in addressing future challenges.

\section{The motivation for development}

Due to legislative, environmental, water availability and operational pressures, as an industry we are moving from simply dealing with tailings 'as is' at the end of mineral processing to engineering the tailings to suit our preferred or required disposal method. Figure 1 illustrates a suggested approach for determination of a waste tailings disposal system (Sofra, 2001).

A common practice in industry is to use the thickener to produce a clear overflow for recycling into the process, with the waste stream produced at the bottom of the thickener being a low concentration Newtonian fluid suspension which is pumped in turbulent flow to a disposal area, usually a large dam. Alternatively, Figure 1 illustrates the case where the disposal area is receiving thickened tailings. Once the tailings is thickened, non-Newtonian characteristics prevail and it becomes essential to understand this behaviour. In a greenfield site it is suggested that the designer works upstream from the preferred method of disposal, through pumping, to the thickener. Compressional rheological characteristics involving the compressive yield stress and the permeability are required to predict, for example, tailings consolidation in the disposal area. Both shear stress-shear rate and yield stress data are required as a first step for pipeline design and start up, for the design of the suction side of the pump and for the design of the thickener, in particular for the torque requirements for the rake and rake drive mechanism.

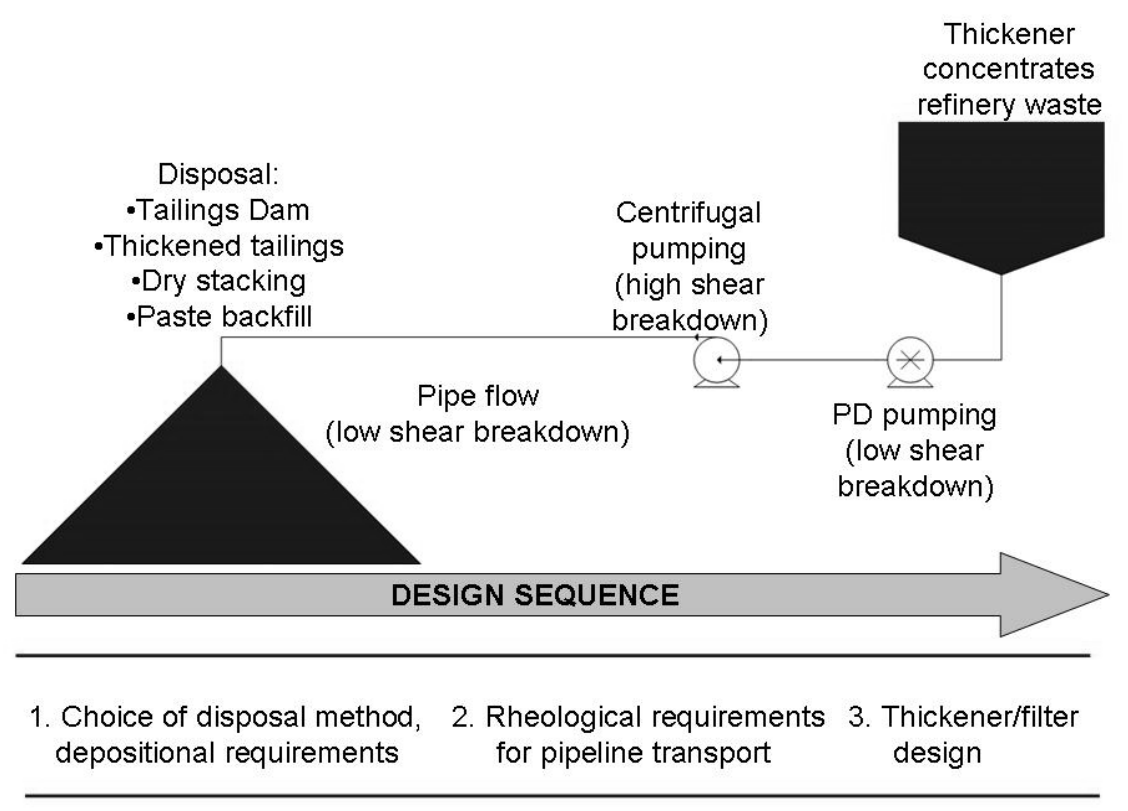

Figure 1 Suggested approach for determination of a tailings disposal system (Sofra, 2001)

In 1974 Boger was approached by Alcoa of Australia with an enquiry about the rheological characteristics of the waste material they called red mud. Due to environmental issues, the desire to recycle reagents and space restrictions, Alcoa was motivated to decrease the volume of highly caustic process water being deposited with the tailings. It was obvious after the meeting that red mud at higher solids concentrations exhibited a change in flow properties with time of shear. It also seemed as if the breakdown in material structure during shear occurred at a more rapid rate than the rebuilding of that structure from rest. In ideal thixotropic behaviour the two timescales are the same and there is no difference between breakdown and structural recovery. The observed red mud behaviour is therefore non-ideal thixotropy but strictly speaking cannot be defined as rheomalaxis either, which is irreversible.

It was presumed that if the structural breakdown could be quantified, it could be exploited by dewatering the tailings to a high concentration and shearing to reduce the viscosity to allow more efficient pipeline transport to the disposal area for drying, consolidation and slow restructuring. To quantify the thixotropic characteristics, a single point measurement was required and this was developed by adopting the vane device 
used in soil mechanics (Nguyen and Boger, 1983, 1985) to measure the yield stress of the material. The yield stress is the stress which must be overcome to cause the material to flow and is the most useful material property for determination of dewatering and transport requirements of thickened tailings and paste systems.

It was later discovered that thixotropy could be exploited in other ways - not just for red mud but for many thickened flocculated slurries and pastes which show a degradation of structure with shear. The thickener can be operated to a higher underflow solids concentration if the underflow (or part of the underflow) is recirculated back into the bottom of the thickener after being sheared in a centrifugal pump. The reduction in the yield stress part of the underflow and injection into the thickener results in a lower overall underflow yield stress which allows the thickener to produce a higher solids concentration without creating rake torque issues or bogging the thickener.

\section{Development of rheological techniques}

Conventional wet disposal involves pumping relatively dilute tailings to a disposal area with a perimeter dam. The dilute slurry normally passes through a clarifier and generally behaves as a Newtonian fluid, with a constant viscosity and no yield stress. In these cases the rheology of the slurry is not of paramount importance and pump sizing and pipeline design data has traditionally been measured using a Marsh funnel, cup and bob rheometer, pipeloop test or capillary rheometer. Settling rates and critical velocities are of more interest as pipe blockage is likely if flow velocities are not sufficient to prevent settling.

However, as fine particle slurries are thickened to higher concentrations, they begin to display nonNewtonian behaviour, with the onset of a yield stress and a viscosity which may vary with shear rate and sometimes shear history. Viscoelasticity is generally of no importance (yet - it may become important in the future as more sophisticated dewatering aids and other additives are used) and the basic measurements required to characterise these materials include the viscosity and how it varies with shear rate and time of shear and the yield stress.

The general definition of the viscosity is shown in Equation (1).

$$
\eta=\frac{\tau}{\dot{\gamma}}
$$

Where $\tau$ is the shear stress and $\dot{\gamma}$ is the shear rate. In order to make the viscosity measurement one must use a device where both the shear stress and the shear rate can be defined. There are four basic flow fields called viscometric flows where the shear stress and the shear rate can be defined and hence one can define the viscosity for a non-Newtonian material. These fields are Poiseuille (tube) flow, Couette (cup and bob) flow, parallel plate and cone and plate (torsion flows). The two most common flow fields are illustrated in Figure 2. Each of these fields has its own significant advantages and disadvantages. Poiseuille flow involves measuring the pressure drop across a long straight tube as a function of flow rate. Pipe loops and capillary rheometers can be designed to make these measurements but the measurements themselves can be labour intensive and often are associated with significant problems at the wall of the tube where slip can occur.

Poiseuille Flow

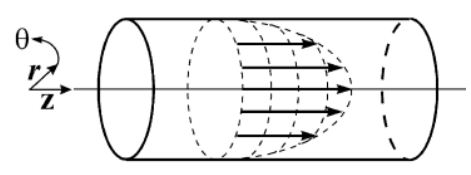

Couette Flow

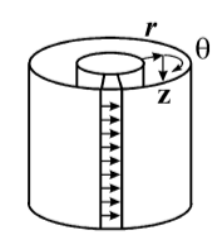

Figure 2 Viscometric flows used for viscosity measurement of non-Newtonian suspensions

The most common geometry used for measurement of shear stress and shear rate is Couette flow using a cup and bob rheometer. This geometry also has a distinct disadvantage for many suspensions in that the gap has 
to be large enough to ensure that the particles themselves do not interfere with the measurement. When the gap is large, the analysis of data becomes complex and often is not understood, especially when yield stress behaviour is evident. Slip is a problem and sedimentation can also occur. Parallel plate torsion flow is very rarely used for measurements of suspensions; again the problems are associated with sedimentation, slip and analysing the raw data. Cone and plate torsion flow, although good for measuring the properties of polymers and polymer solutions, also requires a very small gap for the analysis to be correct. Hence none of the above geometries are ideal for the measurements of the viscous properties of slurries and pastes. Yield stress measurements with conventional rheometric equipment are equally problematic.

\subsection{Yield stress measurement}

Figure 3 illustrates typical shear stress-shear rate behaviour that is observed for a yield stress material. There are very significant misconceptions associated with yielding. Much of the data obtained for suspensions is not obtained at low enough shear rates to determine a true yield stress by extrapolation. Often the data are extrapolated to the ordinate from a linear region of behaviour at high shear rates to define what has been called the Bingham yield stress, $\tau_{\mathrm{B}}$. The Bingham yield stress is a model fitting parameter and has no meaning whatsoever in terms of the true yielding of the material. We have seen many consulting reports in which this yield stress has been used as a basis for design, yet the Bingham yield stress can grossly overestimate the true yield stress as seen in Figure 3, and lead to costly over design. The yield stress is the magnitude of the shear stress at which the material initially flows, and in principal can be determined if the measurements of the shear stress as a function of shear rate are made at low enough shear rates. However, for high concentration slurries, slip regularly occurs in concentric cylinder and capillary geometry at low shear rates. Thus, extrapolated values of the shear stress-shear rate data are not easy to obtain and often involve significant error.

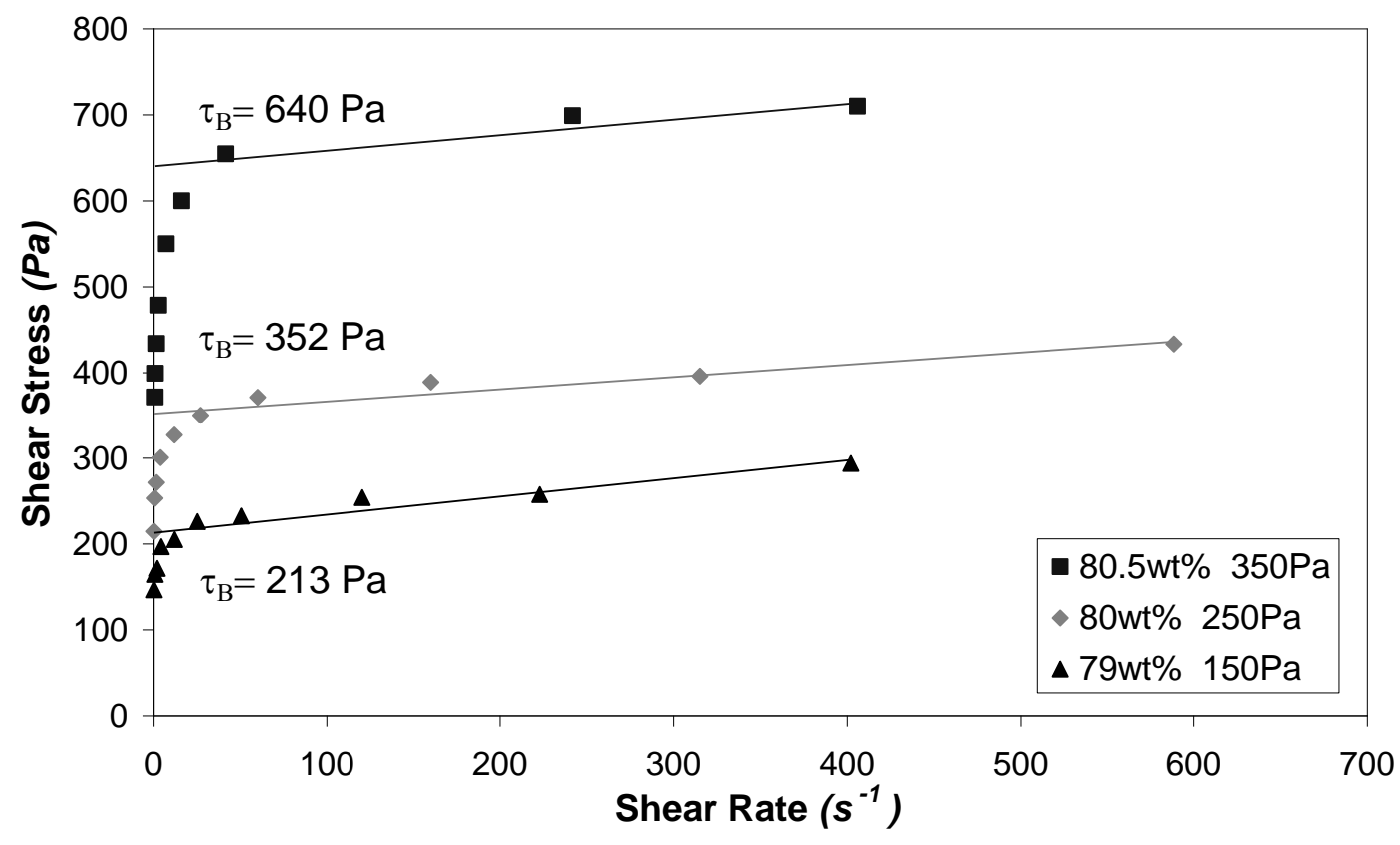
Figure 3 Shear stress-shear rate curve illustrating the over estimation of the Bingham yield stress
compared with the true yield stress

Figure 4 illustrates the further potential errors associated with extrapolation, dependent upon the shear rate region in which data are obtainable. The results in Figure 4 were obtained with a capillary rheometer, a conventional Couette (concentric cylinder rheometer), and a vane in cup device (see Section 3.2.1) used to eliminate slip. Data obtained with the capillary and the Couette rheometers agree at the higher shear rates, but at the lower shear rates both deviate from the vane and cup data which extrapolates to approximately the correct yield stress. At shear rates less than about $300 \mathrm{~s}^{-1}$ (which is significantly greater than the shear rate in most pipelines), both the capillary and Couette data illustrate the dramatic influence of slip flow at a solid surface. At a shear rate of $10 \mathrm{~s}^{-1}$, there is more than a threefold difference in the measured shear stress. 


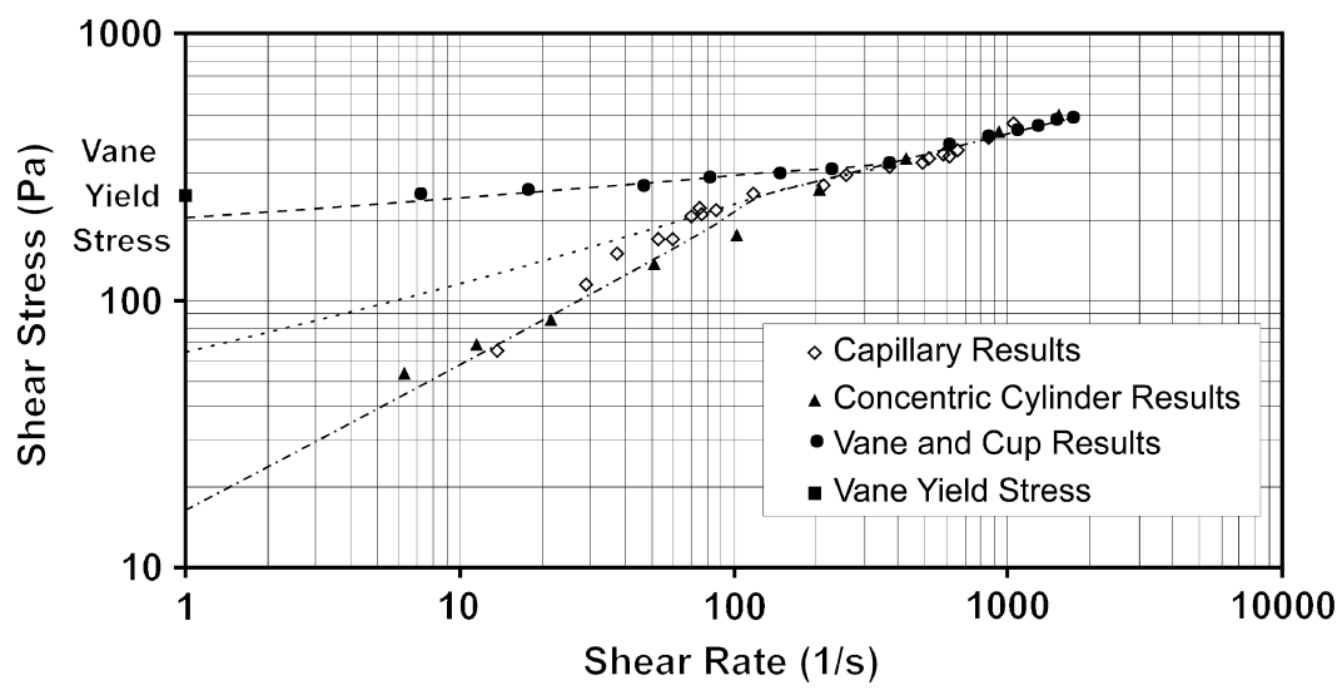

Figure 4 Flow curves for a paste sample $\left(\tau_{\mathrm{y}}=250 \mathrm{~Pa}\right)$. Data are obtained using various geometries

\subsubsection{Yield stress measurement using the vane method}

The true yield stress for the mine stope fill material shown in Figure 4 was obtained using the vane method (Nguyen and Boger, 1983, 1985). The extrapolation of the high shear rate data obtained with a capillary and concentric cylinder device gives a yield stress of $65 \mathrm{~Pa}$, while the extrapolated value obtained from the lower shear rate data is in the order of $18 \mathrm{~Pa}$. The true yield stress was $250 \mathrm{~Pa}$. The errors involved are immense and thus, if the true yield stress is needed, which is the case for rake design in thickeners, for pump re-start and pipeline design, then conventional rheometry geometries should generally not be used and a device like the vane is appropriate.

To summarise the vane method: the vane is inserted into the suspension and rotated at a very low speed $(0.2 \mathrm{rpm})$ where the torque is observed as a function of time. The torque increases until reaching a maximum value, $T_{m}$, when the material yields. The maximum torque is related to the yield stress by Equation (2), where $d$ is the diameter of the vane and $l$ is its height. Equation (2) is valid if end effects are minimised and this is possible by using a large enough $l / d$ vane. The great advantage of the vane is the material yields on itself; slip generally is not an issue and the vane can be inserted into the fluid in different regions to negate any thixotropic effects. This technique is now used worldwide for measurement of yielding in all matter of materials and was motivated by the need generated by the alumina industry.

$$
T_{m}=\frac{\pi}{2} d^{3}\left(\frac{l}{d}+\frac{1}{3}\right) \tau_{y}
$$

\subsubsection{Yield stress measurement using the slump test}

The need for a simple method of measuring the yield stress on site, not requiring a power supply or any sophisticated instrumentation led to the adaptation of the cone slump test used for measuring the consistency of wet cement (Pashias and Boger, 1996). The adapted method involves filling an open-ended cylinder with slurry or paste, lifting the cylinder and measuring the distance the slurry slumps relative to the original container height. The slump is then related to the yield stress by Equation (3) (approximate solution) or Equation (4) (exact solution).

$$
\begin{gathered}
\tau_{y}^{\prime}=\frac{1}{2}+\frac{1}{2} \sqrt{s^{\prime}} \\
s^{\prime}=1-2 \tau_{y}^{\prime}\left(1-\ln \left(2 \tau_{y}^{\prime}\right)\right)
\end{gathered}
$$

$\tau_{y}$, is a dimensionless yield stress $\left(\tau_{y}^{\prime}=\tau_{y} / \rho g H\right)$, and s' is the dimensionless slump which is the actual slump divided by the height of the cylindrical slump vessel, $H$. Numerous direct comparisons of the cylindrical 
slump measurement and the vane yield stress measurement are now available in the literature. One such comparison for different materials from our own work is shown in Figure 5, which illustrates that the yield stress determined from the vane and the slump test are in agreement for engineering design purposes.

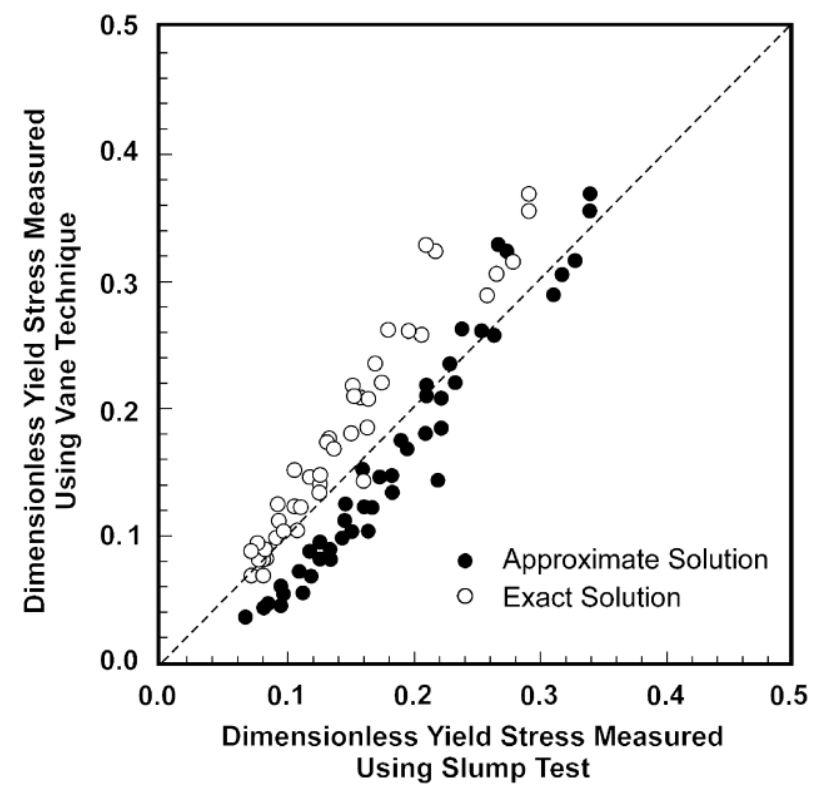

Figure 5 Comparison of the vane and slump yield stress techniques

The slump measurement was again motivated by a need in the alumina industry. Thus the alumina industry and the basic properties of the red mud motivated the development of two methods for single point yield stress measurement which are now used industry-wide and extend well beyond the industry into the wider world. The next task was to find a simple and more accurate method of determining shear stress-shear rate data.

\subsection{Shear stress-shear rate measurement}

The Couette viscometer is most commonly used for obtaining shear stress-shear rate data for many materials and in many industries. The torque on the bob is observed as a function of its rotational speed and these measurements are used to calculate the shear stress and the shear rate. However, calculation of the shear rate depends on knowing a functional form between the shear stress and shear rate, i.e., substituting a fluid model. Such a model will not be available a priori hence approximate techniques are needed to evaluate the shear rate in the cup and bob rheometer, particularly when the gap is wide, which is necessary for paste or suspension-like materials that are of interest here. Often a narrow gap assumption is made in order to define the shear rate. This definition, often embedded in instrument software, is not valid for the wide gap required when dealing with paste-like systems, leading to erroneous results.

\subsubsection{The bucket rheometer (Fisher et al., 2007)}

The shear rate can be calculated from first principles if the outer radius of the cup becomes very large (approaches $\infty$ (Kreiger and Maron, 1954)), or is large enough so that the stress decays to zero prior to the outer radius of the cup. The shear stress and the shear rate on the inner bob surface rotating in an infinite medium can then be directly defined and are not dependent on any particular model assumption. The torque is measured as a function of rotational speed and plotted on a $\log -\log$ graph. Generally, the slope of this graph will be constant, but if not, differentiation of the log-log plot will give the gradient at specific rotational speeds. Once the slope or slope function is known the shear rate can be defined, as can the shear stress, from the torque measurements.

Barnes and Carnalli (1990) first published the idea of using a vane in a cup to minimise slip and a combination of this idea and the infinite medium analysis of Kreiger and Maron has resulted in the 'bucket rheometer' (Fisher et al., 2007; Sofra et al., 2007b). 
Major advantages of using the vane in an infinite medium include the following:

- Insertion of a vane causes less sample disturbance than a cylindrical bob, minimising thixotropic breakdown.

- The vane in cup is less susceptible to errors arising from large particle sizes.

- Yielding occurs between layers of fluid, minimising the effects of wall slip.

- The device is easily portable.

- Shear stress and shear rate are easily determined.

- The analysis is valid for yield stress fluids.

Figure 6 shows data obtained for a nickel laterite pressure acid leach feed slurry. The graph indicates the regions in which data would be required for the design and operation of various unit operations and also illustrates clearly the slip problems associated with cup and bob and the capillary instruments. As is required for low shear rate measurements to be valid, the vane flow curve data extrapolates to the vane yield stress measurement. The vane rheometer is generally not suitable for low viscosity Newtonian fluids (Barnes and Carnelli, 1990).

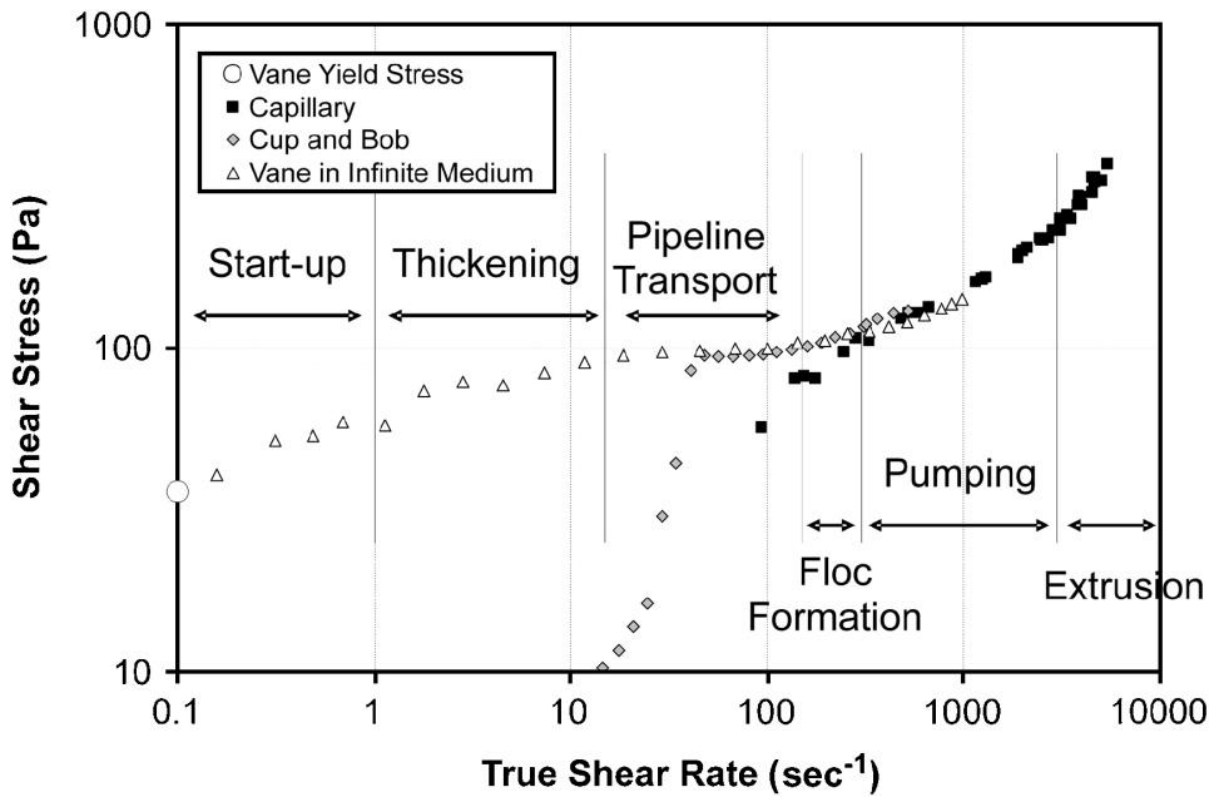

Figure 6 Shear stress-shear rate data for $45.1 \mathrm{wt} \%$ nickel laterite slurry showing the benefits of using the bucket rheometer with vane geometry

\section{$4 \quad$ Rheology as a tool for understanding the effect of physical and chemical factors}

With the ability to reliably measure various rheological phenomena comes the ability to assess the effect of various physical and interfacial chemistry factors on rheology. This information can then be used to either exploit existing conditions or manipulate conditions for optimal processing and tailings disposal.

\subsection{Physical factors}

\subsubsection{Solids concentration}

The response of the rheology to an increase in solids concentration is unique for each system as exemplified in Figure 7, and often varies significantly even within one ore deposit. If one desires to employ a thickened tailings or paste system, it is essential that this unique relationship is well understood. Attempting to operate 
near the 'elbow' of the curve, where the yield stress can vary by $100 \mathrm{~Pa}$ over a solids concentration of only $+/-1 \%$ can be highly problematic due to the inevitable process instabilities that must be considered.

For thixotropic materials, shear history must be taken into account when determining the yield stressconcentration relationship. During the design phase, it is unlikely that the extent of structural breakdown through various unit operations will be known. As such, the yield stress-concentration relationship must be determined for structural states ranging from the (relatively) unsheared state to the equilibrium state. From this data, the operating window can be determined.

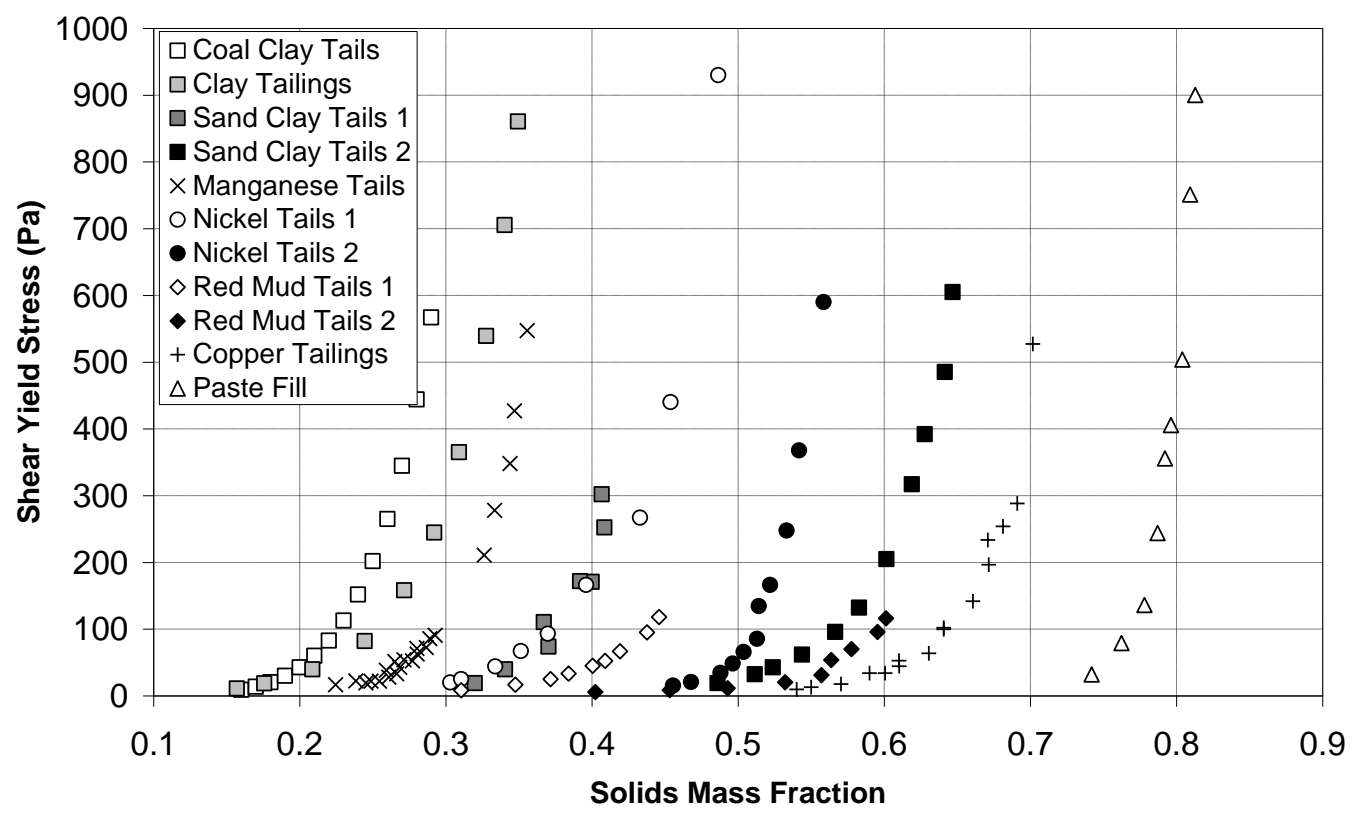

Figure 7 Shear yield stress as a function of solids concentration for a variety of minerals

The most common effect of solids concentration on the viscosity observed in the minerals industry is a shift from Newtonian behaviour at low solids concentrations, through Bingham behaviour at intermediate concentrations to yield stress-shear thinning behaviour at higher concentrations. As with the yield stress, the specific concentration ranges at which these transitions occur is material specific and depends on mineralogy and particle size distribution among other factors.

\subsubsection{Shear rate and shear history}

The effects of shear rate and shear history have been briefly discussed earlier in this paper, but it is worth reiterating that the viscosity of slurries and pastes is very often dependent on the shear rate and as such if a viscosity is quoted then the relevant shear rate must also be quoted. In addition, the shear history should be stated (even if this is only possible qualitatively) for all rheological testwork conducted. Manipulation of shear rates and shear history can provide valuable opportunities for optimising rheological characteristics for a particular application.

It should be noted that not all concentrated slurries are shear history sensitive, nor do they all show a reduction in yield stress and viscosity with time or intensity of shear. Some materials display shear induced aggregation which results in an increase in the structural strength with shear and some friable materials show an increase in the rheology with time of shear due to a reduction in the particle size distribution as shown in Figure 8 . This can become particularly important during long distance pipeline transport. Furthermore, some materials, notably some nickel laterites, show a reduction in rheology when subjected to low shear rates and an increase in the rheology at high shear rates which is reversible if the material is subsequently subjected to low shear rates as shown in Figure 9. This effectively means that the yield stress and viscosity can be increased or decreased at will depending on the shear rate applied (Sofra, 2005). 


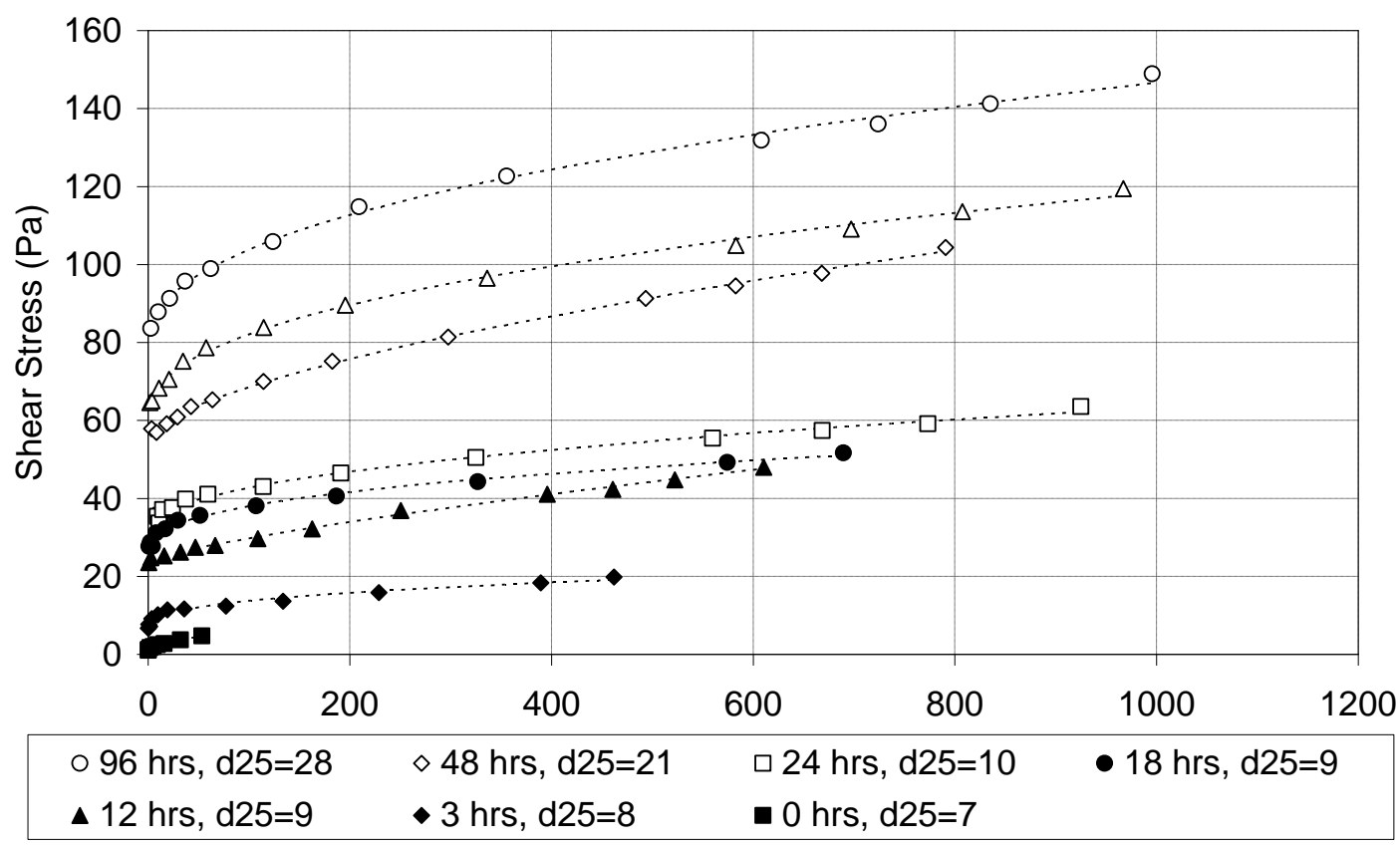

Figure 8 Shear stress versus shear rate as a function of shearing time-bauxite ore

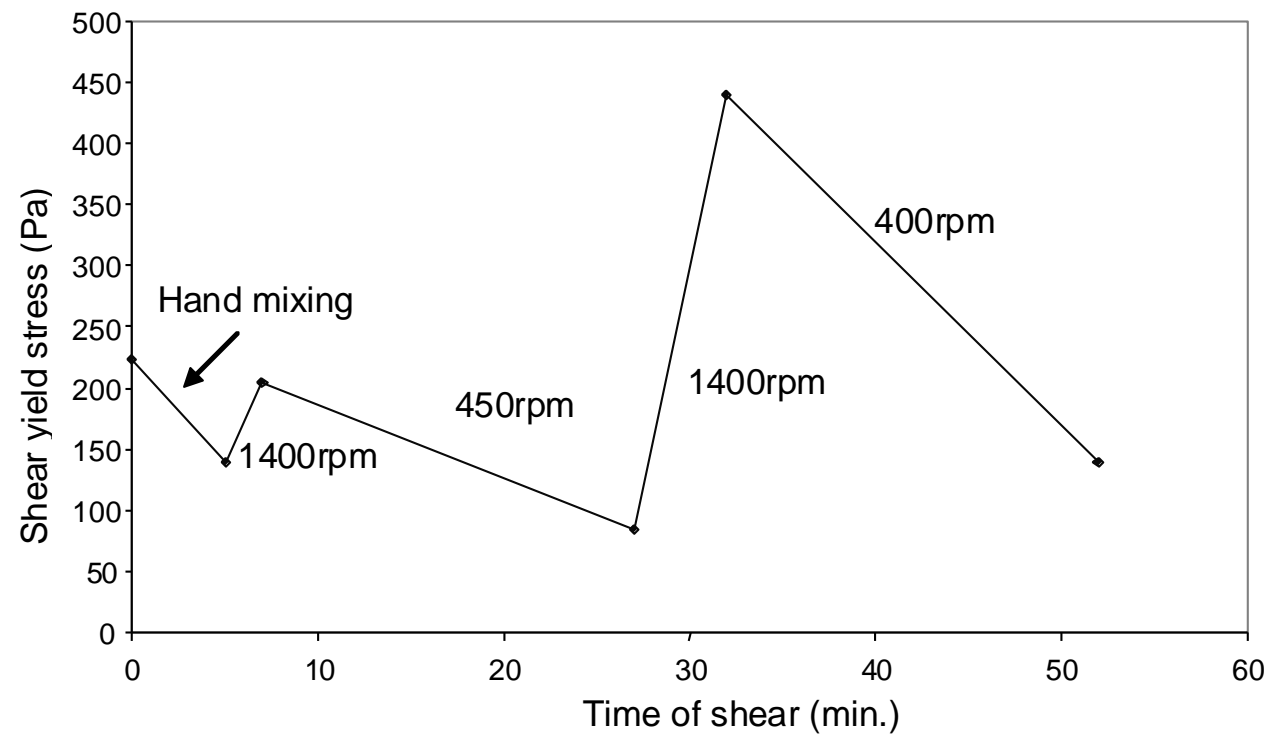

Figure 9 Yield stress progression with varying intensity of applied shear. Nickel Laterite $\mathbf{- 1 5 0} \mu \mathrm{m}$, $60 \mathrm{wt} \%$ solids. Progressive shearing rotational rates (rpm) are indicated

\subsubsection{Particle size distribution}

In general, if all else is equal, a finer grind material will have a higher yield stress and viscosity at a given solids concentration due to the finer slurry having a greater particle specific surface area therefore greater area for interparticle interaction. Finer slurries generally display a more gradual increase in yield stress with increasing solids concentration, whereas coarse materials show a relatively sharp transition from liquid-like to solid-like behaviour as shown in Figure 10.

Knowledge of the effect of particle size and particle size distribution on the rheology is increasingly being exploited to optimise paste backfill operations, where sand is often added to reduce the paste yield stress. 


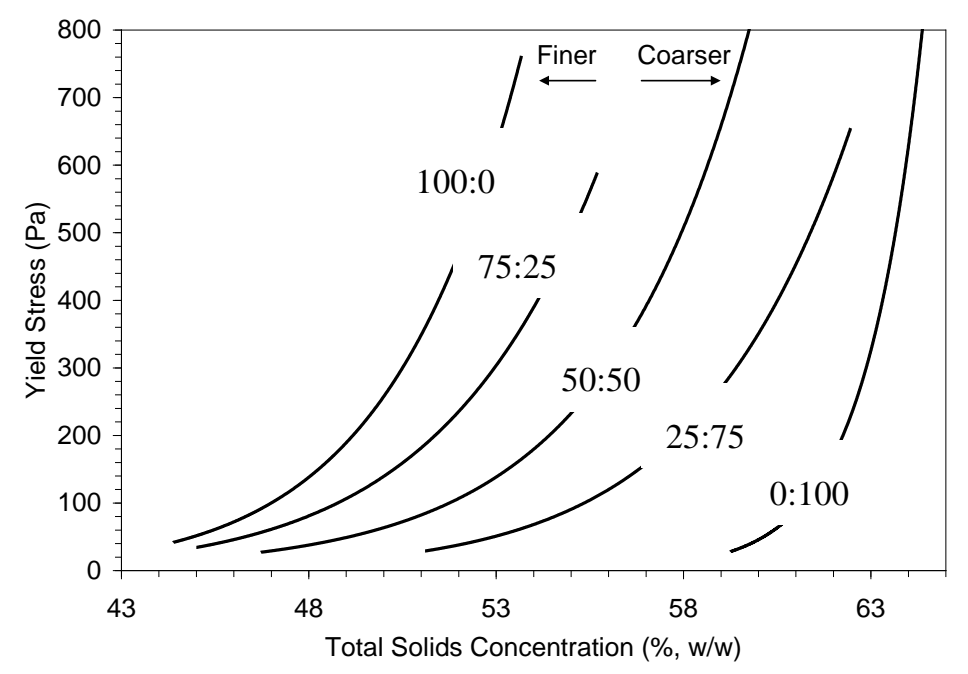

Figure 10 Yield stress profiles for various slimes-sand blends (numbers indicate \%slimes:\%sand)

\subsubsection{Particle shape}

Particle morphology or shape can have a dramatic effect on rheology. This can be particularly noticeable when plate or needle-like particles are present. High aspect ratio particles may show yield stress behaviour and a high shear stress (high viscosity) at low flow rates as the particles are forced to move from random orientation to align in the flow direction. Following this a sudden drop in the shear stress (often to below the yield stress) is observed as the particles become aligned at higher flow rates. This type of behaviour is particularly difficult to analyse, as the resulting flow curve would suggest a negative viscosity due to the decrease in shear stress, which is obviously nonsensical. Nevertheless, it is important to be aware of this behaviour in mixing and agitation applications as it highlights that stagnant zones must be avoided to ensure localised regions of high viscosity do not develop, which would be difficult to remobilise.

\subsection{Interfacial chemistry factors}

\subsubsection{Clays}

Many mineral deposits contain clays in the form of kaolinite, illite, montmorillonite and mixed layer illite/montmorillonite. These clays generally report to the tailings stream for dewatering and storage, are notoriously difficult to dewater and have problematic rheology. Upon addition to water (generally in the initial milling process), some clays hydrate and swell and in many cases, this leads to complete separation or break up of the layers composing the clay.

The dispersion of swelling clays can be controlled by suppressing the swelling via increasing the ionic strength of the aqueous medium to which the clays are added (Van Olphen, 1977; Callaghan and Ottewill, 1974; de Kretser, 1997). As the ionic strength is increased, the electrical double layer is compressed, thereby reducing platelet repulsion and suppressing swelling. If a sufficiently high calcium ion concentration is maintained (lime and gypsum are frequently used), calcium ion exchange will occur with the exchangeable interlayer cations. As calcium is a divalent ion, the neutralising effects will be increased and platelet coagulation will occur at lower concentrations in addition to the promotion of space efficient face-face aggregation. The resulting suspension will have a reduced effective particle surface area, fewer interparticle interactions and a weaker network structure, all of which improve the rheology. Figure 11 highlights the reduction in the yield stress that can be achieved by controlling the dispersion of clays using calcium ions.

The concept of controlled dispersion is not new, however, implementation for tailings disposal is not widespread due to the belief that both clay swelling and complete platelet separation must be avoided, necessitating high calcium concentrations. The high calcium concentrations required for complete controlled dispersion are often not practical due to economic factors and the limited solubility of many available calcium sources leading to excessive scaling issues. However, complete suppression of clay swelling and 
break up is not essential. For improved dewatering performance suppression of break up alone can lead to dramatically improved clay dewatering (Sofra et al., 2007a).

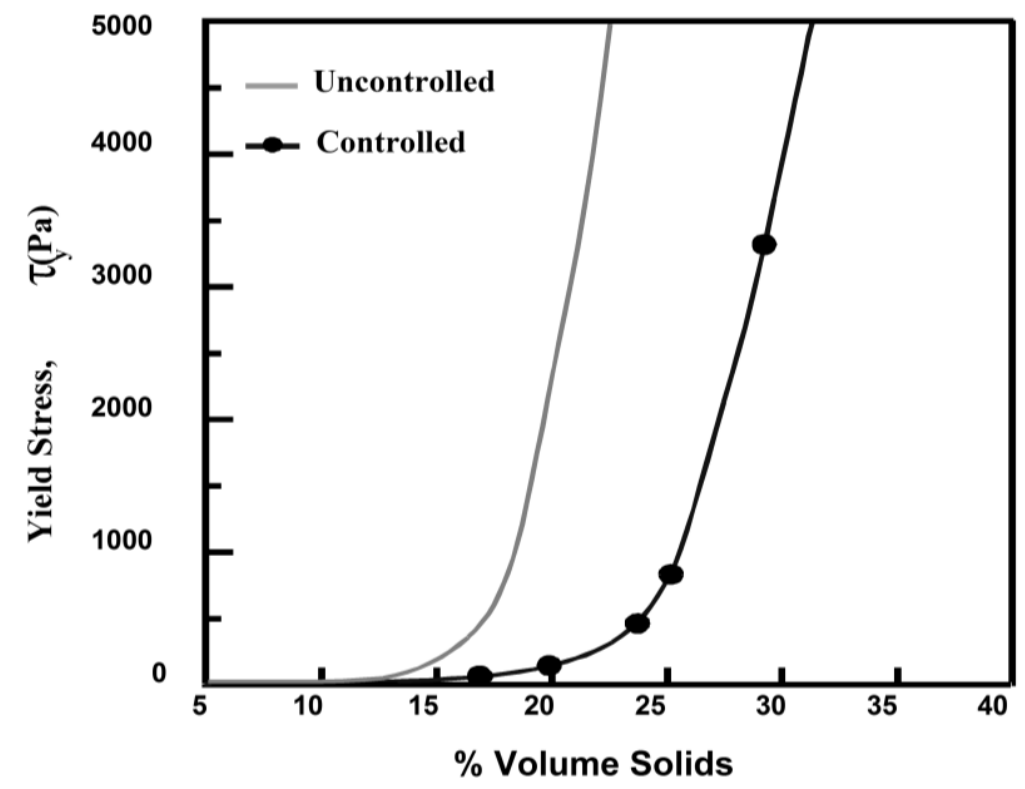

Figure 11 Yield stress-volume fraction curves for controlled and uncontrolled dispersed clay suspensions

\subsubsection{Water chemistry $-\mathrm{pH}$ and Ionic strength}

The trend toward processing ever finer grind slurries has its disadvantages in that the fine particles tend to dominate the rheology, making thickening and pumping more difficult. However, the difficulties with fine suspensions stem from the fact that the finer the particles, the more influential surface chemistry becomes and this can provide opportunities to manipulate the rheology through manipulation of the water chemistry.

Understanding the effect of surface chemistry is achieved by understanding particle surface charge, the effect of surface charge on rheology and how the surface charge can be modified. The surface chemistry can be evaluated by first measuring the zeta potential, which is an indication of the surface charge of the particle as a function of $\mathrm{pH}$. The isoelectric point (iep) can be determined which is the $\mathrm{pH}$ of net zero surface charge and maximum van der Waals attraction. At the iep, the particles are attracted and coagulated and this $\mathrm{pH}$ coincides with a maximum in the yield stress as shown in Figure 12. Away from the iep, where the magnitude of the zeta potential is high, particles are electrostatically repulsed and the system is dispersed, with a low yield stress. It can be seen in Figure 12 that increasing the $\mathrm{pH}$ by one unit can nearly halve the yield stress at high solids concentrations.
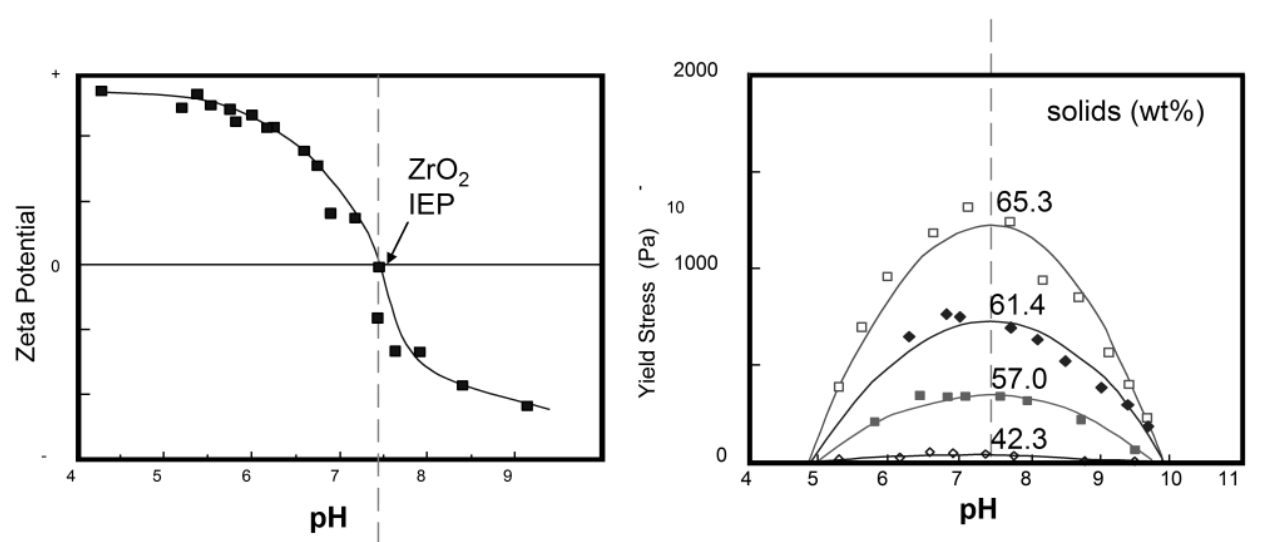

Figure 12 Zeta potential and yield stress curves as a function of $\mathrm{pH}$ for various zirconia suspensions 
Surface interactions and hence the rheology can also be modified by changing the ionic strength of the process water. A high ionic strength compresses the electrostatic double layer of the particle, reducing interparticle repulsion and thus flattens the zeta potential curve as shown in Figure 13. As such, the yield stress becomes less sensitive to changes in $\mathrm{pH}$ than if the ionic strength was low.
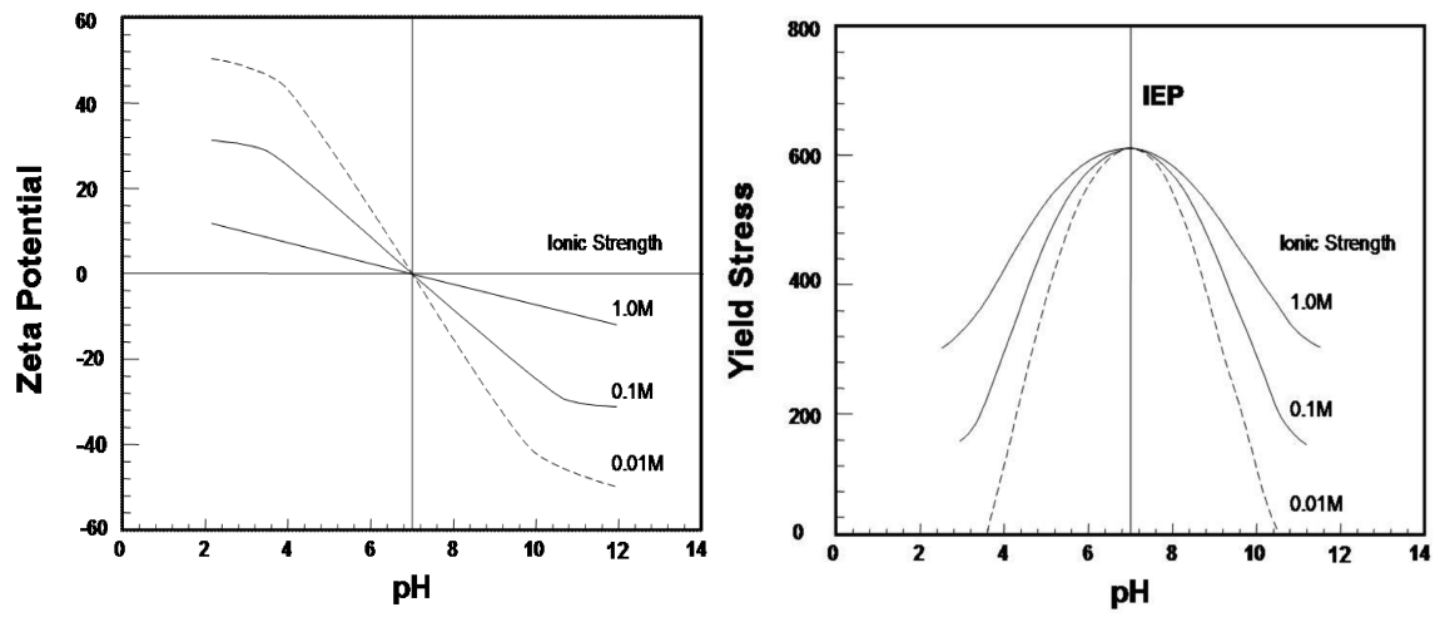

Figure 13 The influence of ionic strength on surface charge and yield stress

This is another example of how advances in rheology have allowed us to measure a physical parameter that provides a very useful link between complex interparticle interactions and how materials behave in real processing situations.

\subsubsection{Additives including flocculants}

Another way in which rheology is being applied is in assessing some of the effects of flocculants and other additives.

It is well understood that flocculants increase the settling rate in a thickener to acceptable levels. However, this is at the expense of the extent of dewatering. Flocculants act by binding fine particles together to form more dense aggregates which settle faster than the individual particles, but water is entrapped within the floc. This water is not fully expelled in the bed of the thickener and the networked structure that is formed in the bed can have a high yield stress.

Rheology is used to determine the effect of shear on flocculated structures and this work is being used to identify and develop flocculants that produce desirable settling rates, create flocs that are robust enough to remain intact during the settling phase but are then easily sheared and compressed in the base of the thickener to allow escape of intrafloc water and a reduction of the yield stress (McFarlane et al., 2006).

\section{Conclusion}

The science of both shear and compression rheology is now an essential tool in the design, operation and maintenance of paste and thickened tailings waste storage schemes. Basic design data can be measured with techniques specifically designed for the purpose. The influence of major variables such as particle size, shape and concentration and the influence of surface chemistry can be evaluated and exploited.

What are the major challenges for the future? A major immediate challenge is in evaluating and exploiting the rheological properties of the new pastes being constructed in high compression thickeners. Constructed is the operable word because the flow characteristics depend on the compression and shear history and often on the time of shear; these materials have a structure. Such flow characteristics were observed in the 1970s with the bauxite residue for the alumina industry in Australia and are the subject of increasing attention. The gellike, high yield stress materials produced (i.e., $\tau_{\mathrm{y}}>200 \mathrm{~Pa}$ ) possess a structure which can be broken down in shear. This structure is dependent on compression history and the structural breakdown is dependent on shear rate and time of shear. In addition the time of structural breakdown and usually particle recovery will depend on time, which is generally not well understood. Thickener manufacturers and suppliers often refer to this 
rheological phenomenon as shear thinning, which describes the phenomenon but does not distinguish it from the rheological term shear thinning which is the simple time independent process of the viscosity is decreasing with increasing shear rate. The immediate challenge is then to understand and exploit this (shear thinning) process which will be compression history dependent.

Another challenge for the future will be assessing the new temperature and shear-sensitive flocculants both in shear and compression, continuing the better understanding of compression rheology in the dewatering process, and recognising that viscoelasticity may be important in high compression flocculated pastes. Understanding the effect of magnetic fields and magnetic particles is also gaining attention in magnetite processing and dense media separation. A major challenge however remains to see more proactive behaviour by regulators and industry in the movement from wet disposal to dry disposal techniques.

\section{References}

Barnes, H.A. and Carnali, J.O. (1990) The vane-in-cup as a novel rheometer geometry for shear thinning and thixotropic materials, Journal of Rheology, Vol. 34(6), p. 841.

Callaghan, I.C. and Ottewill, J. (1974) Journal of the Chemistry Society Faraday Discussions, Vol. 57, p. 110.

De Kretser, R., Scales, P.J. and Boger, D.V. (1997) Improving clay-Based Tailings Disposal: Case Study on Coal Tailings, AIChE Journal, Vol. 43(7), pp. 1894-1903.

Fisher, D.T., Scales, P.J. and Boger, D.V. (2007) The bucket rheometer for the viscosity characterization of yield stress suspensions, Journal of Rheology, Vol. 51(5), p. 82.

Kreiger, I.M. and Maron, S.H. (1954) Direct determination of the flow curves of non-Newtonian fluids, III Standardised treatment of viscometric data, Journal of Applied Physics, Vol. 25(1), pp. 72-75.

McFarlane, A., Bremmell, K. and Addai-Mensai, J. (2006) Improved dewatering behaviour of clay mineral dispersions via interfacial chemistry and particle interactions optimization, Journal of Colloid and Interface Science, Vol. 293, pp. 116-127.

Nguyen, Q.D. and Boger, D.V. (1983) Yield stress measurement for concentrated suspensions, Journal of Rheology, Vol. 27, p. 321.

Nguyen, Q.D. and Boger, D.V. (1985) Direct yield stress measurement with the vane method, Journal of Rheology, Vol. 29, p. 335

Pashias, N. and Boger, D.V. (1996) A fifty-cent rheometer for yield stress measurement, Journal of Rheology, Vol. 40(6), p. 1179.

Robinsky, E.I. (1975) Thickened Discharge - A New Approach to Tailings Disposal, Canadian Mining and Metallurgical Bulletin, Vol. 68, December 1975, pp. 47-53.

Sofra, F. (2001) Minimisation of bauxite tailings using dry disposal techniques, PhD thesis, The University of Melbourne.

Sofra, F. (2005) The importance of understanding feed rheology in nickel laterite processing, in Proceedings Processing Nickel Ores and Concentrates 05, 16-17 November 2005, Cape Town South Africa, MEI, CD-rom only.

Sofra, F., Scales, P.J. and Kilcullen, A. (2007a) Dewatering and clays - the importance of controlling dispersion 'upfront', in Proceedings Tenth International Seminar on Paste and Thickened Tailings (Paste07), A.B. Fourie and R.J. Jewell (eds), 13-15 March 2007, Perth, Australia, Australian Centre for Geomechanics, Perth, pp. 229-238.

Sofra, F., Fisher, D.T. and Boger, D.V. (2007b) The bucket rheometer for thickened tailings and paste flow curve determination, in Proceedings Tenth International Seminar on Paste and Thickened Tailings (Paste07), A.B. Fourie and R.J. Jewell (eds), 13-15 March 2007, Perth, Australia, Australian Centre for Geomechanics, Perth, pp. 249-258.

Van Olphen, H. (1977) Introduction to Clay Colloid Chemistry 2nd ed., Wiley, New York. 
